University of Nebraska - Lincoln

DigitalCommons@University of Nebraska - Lincoln

$12-1-2006$

\title{
A Handheld Neutron-Detection Sensor System Utilizing a New Class of Boron Carbide Diode
}

\author{
Kevin Osberg \\ LSI Logic Corporation, Fort Collins, CO \\ Nathan Schemm \\ University of Nebraska-Lincoln \\ M. Susan Hallbeck \\ University of Nebraska-Lincoln, hallbeck@unl.edu \\ Sina Balkir \\ University of Nebraska-Lincoln, sbalkir2@unl.edu \\ Peter A. Dowben \\ University of Nebraska-Lincoln, pdowben@unl.edu \\ See next page for additional authors
}

Follow this and additional works at: https://digitalcommons.unl.edu/physicsdowben

Part of the Physics Commons

Osberg, Kevin; Schemm, Nathan; Hallbeck, M. Susan; Balkir, Sina; Dowben, Peter A.; Brand, Jennifer I.; and Hoffman, Michael W., "A Handheld Neutron-Detection Sensor System Utilizing a New Class of Boron Carbide Diode" (2006). Peter Dowben Publications. 110.

https://digitalcommons.unl.edu/physicsdowben/110

This Article is brought to you for free and open access by the Research Papers in Physics and Astronomy at DigitalCommons@University of Nebraska - Lincoln. It has been accepted for inclusion in Peter Dowben Publications by an authorized administrator of DigitalCommons@University of Nebraska - Lincoln. 


\section{Authors}

Kevin Osberg, Nathan Schemm, M. Susan Hallbeck, Sina Balkir, Peter A. Dowben, Jennifer I. Brand, and Michael W. Hoffman 


\title{
A Handheld Neutron-Detection Sensor System Utilizing a New Class of Boron Carbide Diode
}

\author{
Kevin Osberg, Nathan Schemm, Sina Balkır, Jennifer I. Brand, M. Susan Hallbeck, \\ Peter A. Dowben, and Michael W. Hoffman
}

\begin{abstract}
A handheld neutron-detection sensor application is described in this paper. The sensor system utilizes a new class of boron carbide diode that interacts with incoming neutrons. To interface with the boron carbide diode, an integrated front end is designed in a $1.5-\mu \mathrm{m}$ standard CMOS technology. With the diode and front-end microchip, a handheld neutron-detection system was realized with an embedded microcontroller for real-time processing. The handheld detector operation was then tested with a plutonium-beryllium neutron source. Test and measurement results confirm the validity of the approach and the functionality of the design.
\end{abstract}

Index Terms-Boron carbide, charge-sensitive amplifier, heteroisomeric diode, neutron detection.

\section{INTRODUCTION}

A COMPACT, reliable, and accurate neutron-detection system may have many different potential applications. In spite of the technical difficulties, neutron-emitting fissionable materials can be detected with solid-state detectors. Systems no larger than a cigarette pack could be used to screen shipping containers for hazardous or illegal radioactive materials and some interdiction is potentially possible. With detectors of sufficient area, baggage could quickly be screened at airports around the world. The monitoring of legacy nuclear weapons stockpiles, reactors, and stored materials in remote locations could also greatly benefit from compact low-power solid-state detection systems. Existing commercial neutron-detection instruments are not practical for such applications. The larger and less reliable gas and liquid neutron detectors currently available

Manuscript received November 13, 2005; revised January 19, 2006 and January 20, 2006. This work was supported by the National Science Foundation through Grant ECS-0300018, the Office of Naval Research through Grant N00014-04-1-0605, the Nebraska Research Initiative, and the U.S. Department of Energy National Nuclear Security Administration Office of Nonproliferation Research and Engineering (NA-22) through the Pacific Northwest National Laboratory. The associate editor coordinating the review of this paper and approving it for publication was Prof. Eugenii Katz.

K. Osberg is with LSI Logic Corporation, Fort Collins, CO USA (e-mail: kosberg@bigred.unl.edu).

N. Schemm, S. Balkır, and M. W. Hoffman are with the Department of Electrical Engineering, University of Nebraska-Lincoln, Lincoln, NE 68588-0511 USA (e-mail: nschemm@bigred.unl.edu; sbalkir@unl.edu; mhoffman1@unl.edu).

J. I. Brand is with the College of Engineering and Technology, University of Nebraska-Lincoln, Lincoln, NE 68588-0511 USA (e-mail: jbrand@unl.edu).

M. S. Hallbeck is with the Department of Industrial and Management Systems Engineering, University of Nebraska-Lincoln, Lincoln, NE 68588-0518 USA (e-mail: hallbeck@unl.edu).

P. A. Dowben is with the Behlen Laboratory of Physics, Department of Physics and Astronomy, University of Nebraska-Lincoln, Lincoln, NE 68588-0111 USA (e-mail: pdowben@unl.edu).

Digital Object Identifier 10.1109/JSEN.2006.883905 are bulky, expensive, and easily damaged, and they require large amounts of power.

Many existing and proposed solid-state neutron detectors also suffer from very low efficiencies and are not suitable for harsh environments [1]. However, recent work has resulted in a new and unique class of solid-state boron carbide semiconductors, which possesses excellent neutron-detection properties [2]-[4]. These boron carbide diodes have been observed to accurately detect single neutrons and potentially provide very high detection efficiencies. Boron carbide also has excellent mechanical properties, making it resistant to harsh environments.

A solid-state handheld neutron-detection sensor system utilizing this new class of diode is presented in this paper. This paper is outlined as follows: In Section II, the properties and operation of boron carbide diodes are described. Section III presents a custom-designed and -fabricated integrated charge amplifier circuit and other components as a sensor front end. The design and realization of the handheld neutron detector is also presented in this section. Test and measurement results that demonstrate the validity of the presented approach are presented in Section IV. Finally, Section V concludes this paper.

\section{Boron CARbide Neutron Detector Diode}

\section{A. Background}

Boron is a useful element for neutron detection. The stable isotope ${ }^{10} \mathrm{~B}$ has high cross section (approximately 3800 barns) for neutrons at lower energies $(\sim 25 \mathrm{MeV})$ based on the ${ }^{10} \mathrm{~B}_{(n, \alpha)}{ }^{7} \mathrm{Li}$ capture, giving

$$
\begin{array}{r}
{ }^{10} \mathrm{~B}+n \rightarrow{ }^{7} \operatorname{Li}(0.84 \mathrm{MeV}) \\
+{ }^{4} \mathrm{He}(1.47 \mathrm{MeV})+\gamma(0.48 \mathrm{MeV}) \\
{ }^{10} \mathrm{~B}+n \rightarrow{ }^{7} \operatorname{Li}(1.02 \mathrm{MeV})+{ }^{4} \mathrm{He}(1.78 \mathrm{MeV})
\end{array}
$$

with $94 \%$ and $6 \%$ probabilities, respectively [5], [6]. Thus, an incoming epithermal (very low energy) neutron strikes a ${ }^{10} \mathrm{~B}$ atom, producing ${ }^{7} \mathrm{Li}$ and ${ }^{4} \mathrm{He}$ capture products, which can generate a substantial amount of electron-hole pairs due to their significant kinetic energies (generally $0.84 \mathrm{MeV}$ for $\mathrm{Li}$ and $1.47 \mathrm{MeV}$ for $\mathrm{He}$ ). Natural boron is about $20 \%{ }^{10} \mathrm{~B}$ and about $80 \%{ }^{11} \mathrm{~B}$, but enrichment of the ${ }^{10} \mathrm{~B}$ is certainly possible.

Previously reported neutron detector devices can be classified as either conversion layer or heterojunction diodes. In conversion-layer devices, a weakly conducting boron-rich layer 
is placed adjacent to a conventional semiconductor diode, and a portion of the charge tracks of the daughter fragment $\mathrm{Li}$ and $\mathrm{He}$ is then detected in the conventional diode (usually $\mathrm{Si}$ or a GaAs diode) [5], [6], [7]-[12]. The heterojunction diode contains boron in a boron carbide layer only on one side (typically the p-layer) and is somewhat similar in structure [2][4], [7], [13]. However, the boron-rich layer is a semiconductor, which is an integral part of the sensor diode. As described in [2], [4], and [7], both the heterojunction-diode and conversionlayer devices have pulse height spectra characteristic of strong contributions from $0.84 \mathrm{MeV}{ }^{7} \mathrm{Li}$ and $1.47 \mathrm{MeV}{ }^{4} \mathrm{He}$, with weaker contributions from $1.02 \mathrm{MeV}{ }^{7} \mathrm{Li}$ and $1.78 \mathrm{MeV}{ }^{4} \mathrm{He}$ [2], [4]-[13], as shown in the capture reactions (1) and (2).

A key factor in efficient neutron detection is the ability of materials to capture the neutrons and provide a semiconductor suitable to both detect and transmit the signal without losses. Semiconducting boron carbides are a new class of materials that are multifunctional in precisely this way, making them ideal for applications such as neutron detection and radioactive decay calorimetry [2], [4], [7], [13]-[16]. As a new category of neutron detector device, the all boron carbide diode has been recently reported in [14] and [15]. The all boron carbide diode offers much improved detection efficiencies; consequently, there has been recent interest in boron carbide based neutron detectors [5], [6], [17].

\section{B. The All Boron Carbide Diode}

The all boron carbide is an entirely new class of diode called the "heteroisomeric diode," where the n-type and p-type semiconducting materials are made from molecules that are identical in formula but different in atomic arrangement (isomers). This is not quite a homojunction diode as the n- and p-layers differ in polytype, but this is not a heterojunction either, as the two semiconductor layers are still very similar semiconductors. The conventional homojunction diode is made with one type of semiconductor (usually $\mathrm{Si}$ or GaAs) where doping is used to establish the majority carriers, i.e., holes in the p-layer and electrons in the n-layer, to form the diode. The heterojunction diode is formed typically from dissimilar materials: ZnSe/GaAs or even $\mathrm{B}_{5} \mathrm{C} / \mathrm{Si}$ [2] or $\mathrm{B}_{5} \mathrm{C} / \mathrm{SiC}$ [3].

The heteroisomeric diode is formed from boron carbides with only subtle differences in structure (largely the relative placement of carbon) [14]. Here, the p- and n-type boron carbide layers were deposited by plasma-enhanced chemical vapor deposition (PECVD) from two different isomers of closodicarbadodecaborane, namely 1) closo-1,2 dicarbadodecaborane (orthocarborane, $\mathrm{C}_{2} \mathrm{~B}_{10} \mathrm{H}_{12}$ ) and 2) closo-1,7 dicarbadodecaborane (metacarborane, $\mathrm{C}_{2} \mathrm{~B}_{10} \mathrm{H}_{12}$ ), which differ only by the carbon position within the icosahedral cage. The PECVD is similar to that described for both heterojunction [2], [3], [18], and homojunction diodes [19] of boron carbide. The boron carbide semiconductor films formed are clearly self-doping materials, since the deposition and decomposition involves only the metacarborane and orthocarborane source molecules (n-type and p-type, respectively) [14]. Fig. 1 shows the conceptual diagram of a heteroisomeric diode, the source molecules that are decomposed to make the diode layers, and a
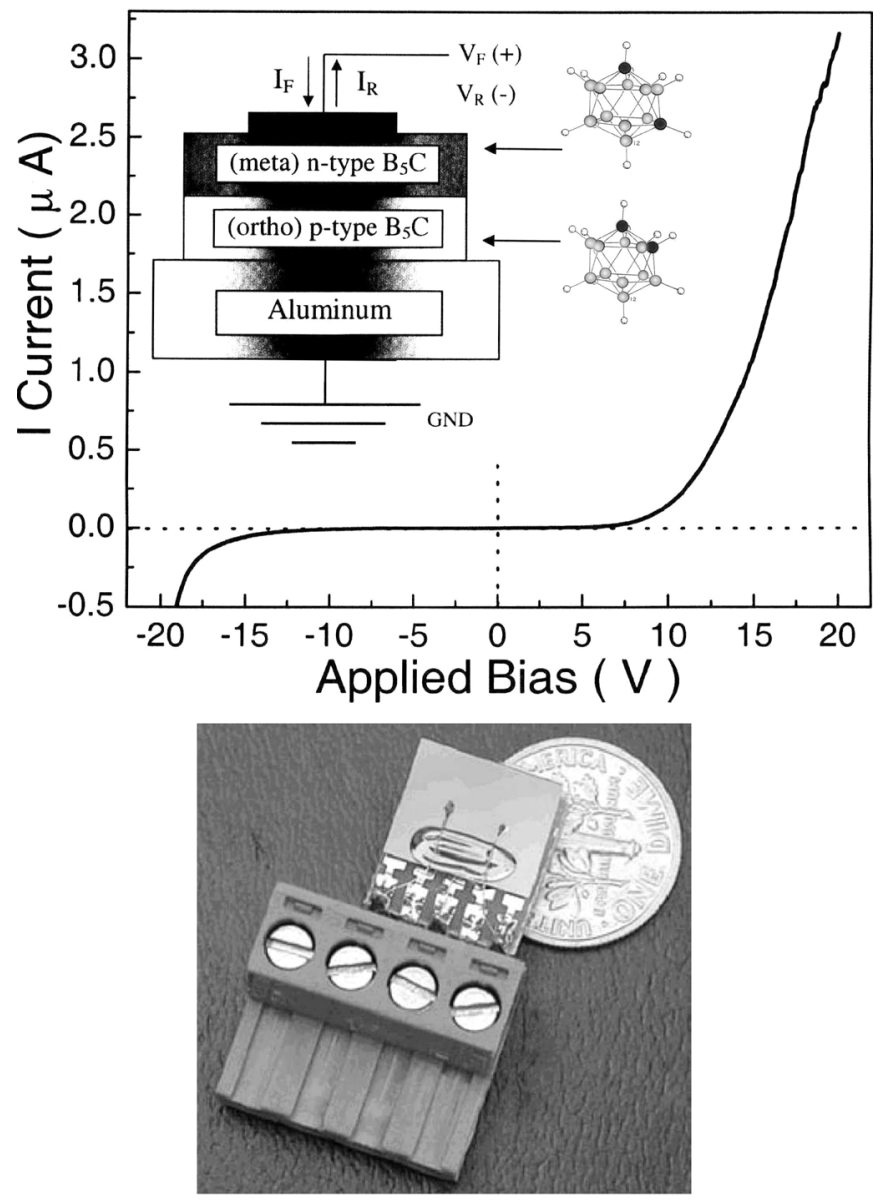

Fig. 1. Heteroisomeric diode conceptual diagram (top) and picture of a fabricated diode sample (bottom). The diode measures approximately $1 \mathrm{~cm} \times 1 \mathrm{~cm}$ in size, and it is connected to a terminal block with wires extending from the aluminum bottom plate (ground connection) and n-type layer (bias and signal connection).

fabricated sample. The $I-V$ characteristic of a diode suitable for neutron detection is also displayed in this figure.

To model thermal neutron detection for the all boron carbide device, constant straight paths were employed for the ${ }^{7} \mathrm{Li}$ and ${ }^{4} \mathrm{He}$ capture products, which are assumed to be emitted isotropically in opposite directions [20], [21]. These capture products lose kinetic energy along these paths, leading to charge pulse production. The probabilities for capture of a normally incident thermal neutron within a $2-\mu \mathrm{m}$-thick boron carbide layer result in deposition of energies [22]. The general decrease of capture probabilities with increasing energy deposition, punctuated by sum pulse height peaks at 2.31 and $2.8 \mathrm{MeV}$, corresponding to capture reactions ${ }^{10} \mathrm{~B}+n \rightarrow{ }^{7} \mathrm{Li}+{ }^{4} \mathrm{He}+\gamma$ and ${ }^{10} \mathrm{~B}+$ $n \rightarrow{ }^{7} \mathrm{Li}+{ }^{4} \mathrm{He}$, respectively, are attributable to the geometry of the device [20], [21]. These expectations were largely observed in the neutron-detection experiment that employs the heteroisomeric diode, which are discussed in Section IV.

Boron carbide diodes are the first real-time solid-state neutron detectors. Experimental current pulses in the range of hundreds of nanoamperes with $10-40-\mu$ s durations result from pulses with charge contents as large as $10^{6}$ electron-hole pairs generated per neutron. With very high collection rates of the electron-hole pairs and operation from at least $20{ }^{\circ} \mathrm{C}-350{ }^{\circ} \mathrm{C}$, 


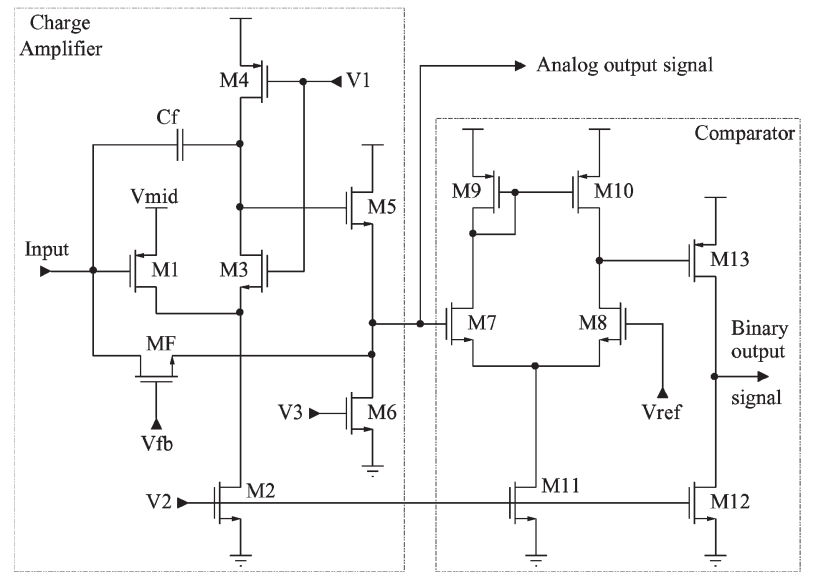

Fig. 2. Circuit diagram of the charge amplifier and comparator used in the integrated sensor front end.

these detectors are potentially more efficient and reliable than any other neutron-detecting semiconductor reported to date [4].

\section{Sensor System Design}

A handheld neutron detector that utilizes the aforementioned boron carbide diode is described in this section. The handheld is composed of two main modules, namely 1) a sensor front end and 2) a microcontroller-based section. The sensor front end is a charge-sensitive amplifier that converts the charge pulses from neutron-detection events to detectable voltage levels. The amplifier is essentially a lossy integrator with a feedback capacitor bank for charge-to-voltage conversion. The microcontrollerbased section acts as a processing and control module for the overall handheld. These modules are presented next.

\section{A. Sensor Front End Microchip}

The ability of the charge amplifier to convert small charge signal inputs to a voltage signal make it particularly useful as a particle-detector interface [23], [24]. The low-noise charge amplifier used here as the neutron detector front end was first presented in [25] and [26]. The topology of the amplifier is shown in Fig. 2 and was designed and fabricated in a $5-\mathrm{V} 1.5-\mu \mathrm{m}$ standard CMOS process. The core of the charge amplifier consists of the folded-cascode pair M1 and M3 and active load M4. The cascode structure provides the charge amplifier with a high open-loop gain and large frequency bandwidth. The output of the folded-cascode pair is then buffered with a source follower created by transistors M5 and M6. The source follower then biases the input through feedback transistor MF.

Without the feedback transistor and capacitor, the open-loop gain $A_{0}$ is given by

$$
A_{0} \approx-g_{m 1}\left(\left(r_{\mathrm{ds} 1} g_{m 3} r_{\mathrm{ds} 3}\right) / / r_{\mathrm{ds} 4}\right)
$$

where $g_{m 1}$ and $g_{m 3}$ are the transconductances of M1 and M3, respectively, and $r_{\mathrm{ds}}$ is the drain-to-source resistance of the MOS devices. Note that the open-loop gain is dominated by the drain-to-source resistance $r_{\mathrm{ds} 4}$ of $\mathrm{M} 4$, which is in parallel with the output impedance of the folded-cascode pair as shown in (3). The source follower pair M5 and M6 is approximated as unity gain in (3). With feedback capacitor $C_{f}$, the ideal closed-loop form of the charge-to-voltage conversion gain $A$ is given by

$$
|A| \approx \frac{1}{C_{f}} .
$$

The boron carbide diodes are placed under reverse bias during detection and are capacitively coupled to the chargesensitive amplifier. Hence, charge pulses resulting from neutron capture events flow from the input to ground, causing the stored charge in $C_{f}$ to change, and vary the voltage at the input node. That small voltage change is then amplified at the output as a positive-going voltage peak. A controllable feedback resistor implemented by MF then causes the output voltage to decay to a steady-state dc value and will prevent the amplifier from saturating due to closely arriving charge transfers.

To create a digital pulse signal from the charge amplifier and provide a degree of noise rejection, a simple comparator is implemented. The comparator circuitry is also shown in Fig. 2 and consists of an n-type differential pair amplifier coupled with a p-type common-source amplifier. An n-type differential pair is used so that the dc output level of the charge amplifier can properly bias input transistor M7. The addition of the common-source amplifier provides a near rail-to-rail operation, and additional CMOS inverters provide a fully digital output. The operation of the comparator provides a logic high while the charge amplifier output exceeds $V_{\text {ref }}$ and a logic low while less than $V_{\text {ref }}$. Thus, the comparator output can be used as a strobe signal to count neutron-detection events.

Since the comparator design is a simple two-stage amplifier, it will suffer from an input-offset voltage. However, in this application, the resolution of small signal inputs is not required. Instead, to suppress noise-induced false detections, $V_{\text {ref }}$ is often on the order of hundreds of millivolts above the dc level of the charge amplifier.

In order to fully tune the integrated circuitry, all bias voltages were generated off-chip. In addition, feedback capacitor $C_{f}$ of Fig. 2 was realized through the use of different on-chip poly1/poly 2 capacitors. Four capacitors were created with ideal values of $1.5,3.5,10$, and $20 \mathrm{pF}$. These capacitors can then be connected as $C_{f}$ through externally controlled switches. At the same time, multiple capacitors can be connected in parallel, providing a wide range of values for controlling the conversion gain. The die photo of Fig. 3 shows the selectable bank of capacitors, charge amplifier, and two comparators that occupy an active area of $880 \mu \mathrm{m} \times 880 \mu \mathrm{m}$. For added flexibility, the second comparator is present on the die to facilitate the comparison of the waveforms with two different reference voltages simultaneously.

\section{B. Handheld Neutron Detector}

With the integrated front end interfacing with the boron carbide diode, a standalone handheld neutron detector was designed and realized around an Atmel AT91 microcontroller. The microcontroller samples the charge amplifier analog output 


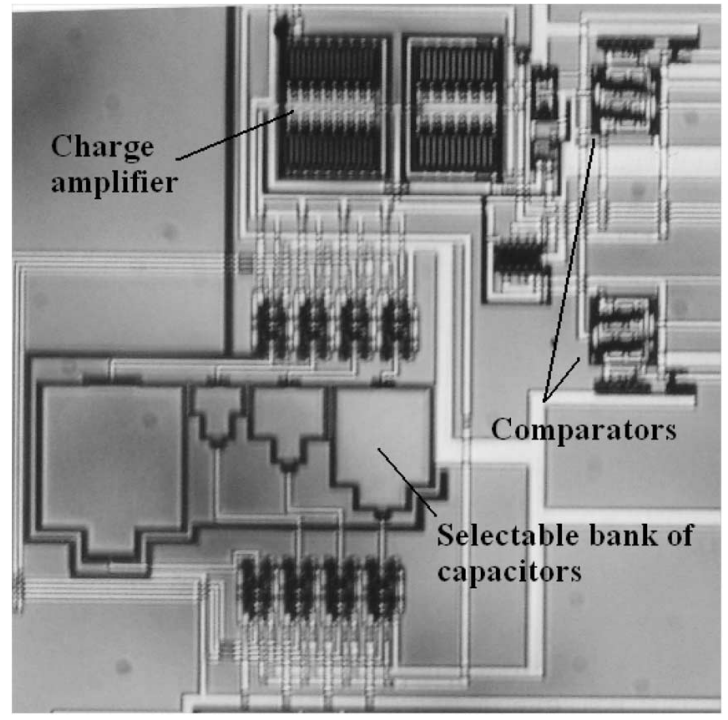

Fig. 3. Die photo of the integrated front end showing the charge amplifier, comparators, and configurable bank of capacitors.

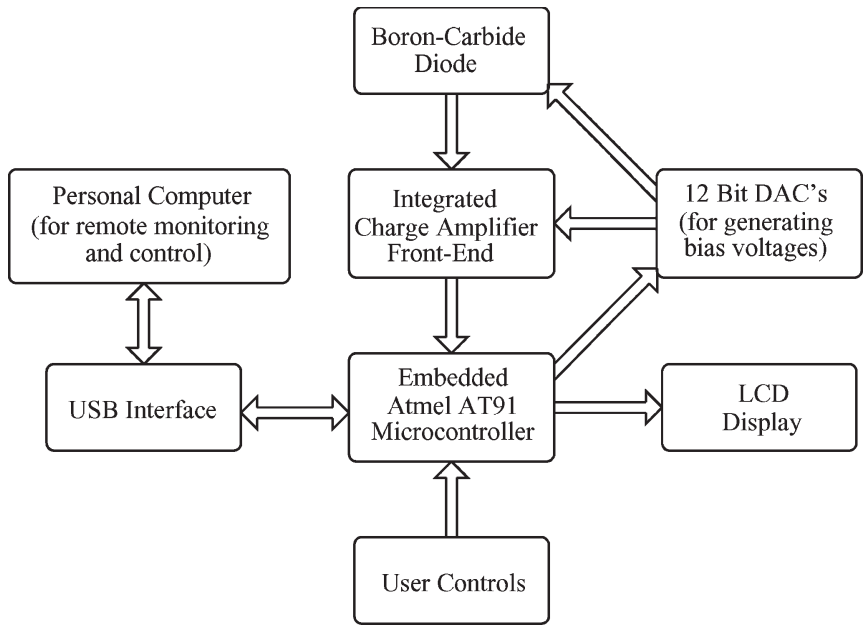

Fig. 4. Architecture and control flow for the design of handheld neutron detector.

at $1.3 \mathrm{Msamples} / \mathrm{s}$ with a 12-bit resolution and performs realtime processing on the waveform to provide information about the energy of each neutron capture event. A universal serial bus (USB) interface and custom software modules allow the handheld system to interface with a computer for capturing, displaying, and downloading results. The microcontroller also provides programmable bias voltages for the front-end chip through 12-bit digital-to-analog converters. A simplified architecture and control flow diagram for the design of the detector is shown in Fig. 4. The circuit board containing the microcontroller and sensor front-end chip can be seen in Fig. 5. The circuit board was also packaged with a 3.6-V NiMH rechargeable battery, LCD, and simple button interface for navigating display menus and adjusting the bias voltages of the front end chip. Fig. 5 (bottom) shows the entire handheld with a boron carbide diode connected to the sensor port and USB cable connection. The overall package of the detector is comparable in size to a handheld personal digital assistant with a standby time of more than $24 \mathrm{~h}$ and continuous operation time of $10 \mathrm{~h}$.

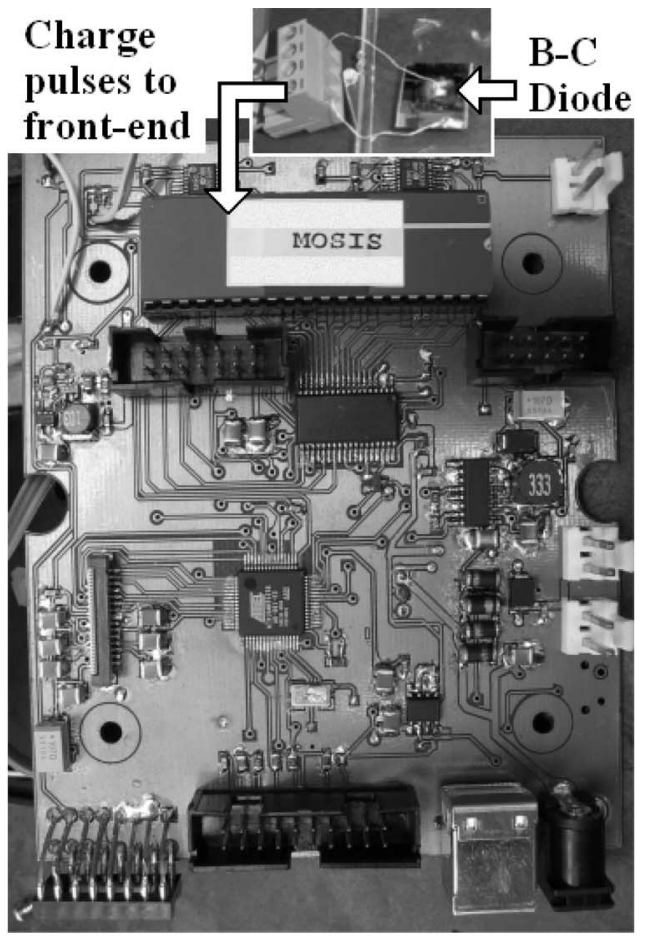

(a)

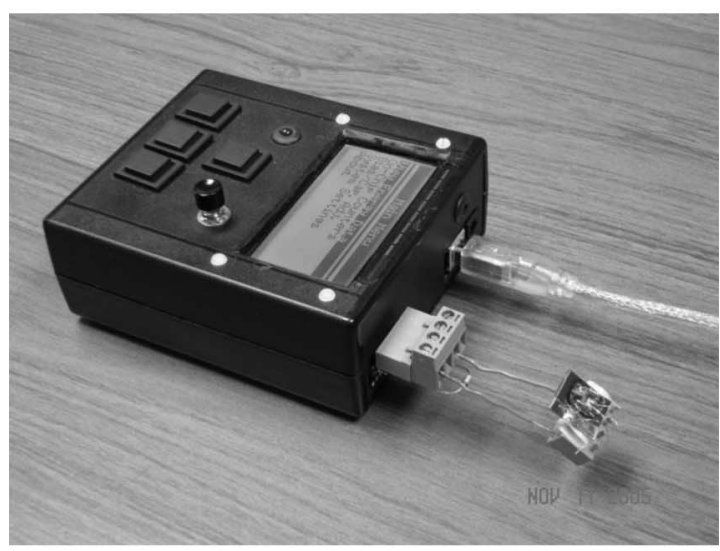

(b)

Fig. 5. Custom-designed circuit board (top) for handheld neutron detector containing the front-end microchip (located at top) and microcontroller (located in the middle). A boron carbide diode is also pictured. The charge pulses generated by the sensor diode based on neutron capture events are processed by this circuit board inside the handheld. The microncontroller drives the liquid crystal display (LCD), provides the USB computer interface, controls the frontend chip, and samples the pulses for processing. The entire handheld with sensor and USB cable connection is also shown (bottom).

\section{EXPERIMENTAL RESUlTS}

The operation of the sensor front end section was first tested with inputs simulating the charge pulses of the boron carbide diode. A simple $R C$ differentiator circuit was connected to the charge amplifier input through a coupling capacitor. The negative edges of a square-wave input to the differentiator create current pulses flowing in the correct direction. The charge content of the pulses can be controlled by the amplitude of the wave. The resulting output waves for one input falling edge are shown in Fig. 6. The top waveform is the voltage spike created by the charge amplifier. In this case, the integrated capacitors were configured to provide a $5-\mathrm{pF}$ feedback capacitance. Based 


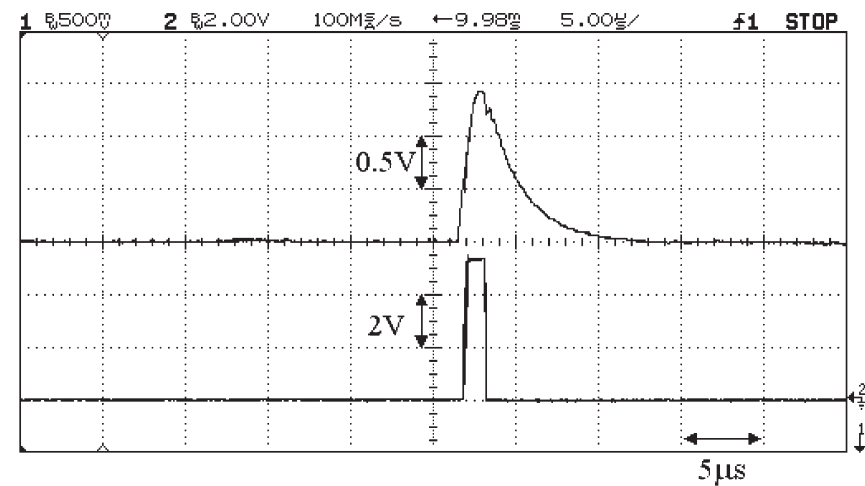

Fig. 6. Oscilloscope captured waveforms of the charge amplifier (top) and comparator (bottom) waveforms created from simulated neutron input.

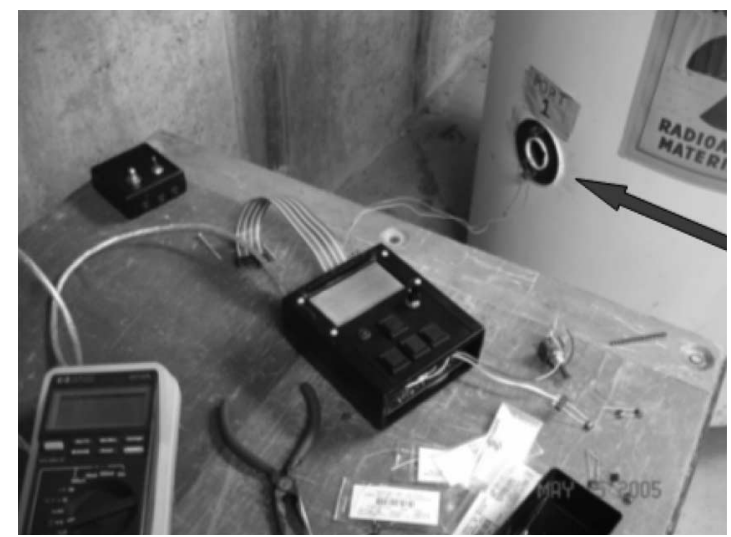

Fig. 7. Testing of the handheld neutron detector with a plutonium-beryllium neutron source. Radiation chamber insertion point for the neutron source is indicated by arrow.

on (4), the ideal gain should be $200 \mu \mathrm{V} / \mathrm{fC}$. The measured gain based on the differentiator circuit and charge amplifier output is approximately $170 \mu \mathrm{V} / \mathrm{fC}$. The slightly lower measured gain is due to the time constant of the charge amplifier feedback, which is comparable to the input pulse width. The bottom waveform of Fig. 6 is the comparator output, which shows the digital pulse created while the charge amplifier output exceeds $V_{\text {ref }}=$ $2.9 \mathrm{~V}$. Neutron testing was then conducted with a paraffinmoderated plutonium-beryllium neutron source. Fig. 7 displays the handheld next to the neutron source during testing. The boron carbide diode was placed inside the radiation chamber 1 in away from its center with an extended cable in order to receive the calibrated neutron flux rate. The surface area of the diode used in the experiment was approximately $1 \mathrm{~cm} \times 1 \mathrm{~cm}$. Also shown in this figure are various cables extending out of the handheld for testing purposes. Calculations on the source estimate a neutron flux rate of approximately 20 neutrons $/ \mathrm{ms}$ over a $1 \mathrm{~cm} \times 1 \mathrm{~cm}$ area. A $15-\mathrm{pF}$ capacitor setting for the charge-sensitive amplifier was used during the measurements reported here, and the boron carbide diode was reverse-biased at $3 \mathrm{~V}$. A typical charge amplifier output waveform captured over a period of $10 \mathrm{~ms}$ by the sensor system is shown in Fig. 8. The waveform shows several large spikes resulting from neutron detection and confirms the output of the charge amplifier seen in Fig. 6. In this example, the average number of counts observed above a threshold of $1 \mathrm{~V}$ is 10 over a 10 -ms

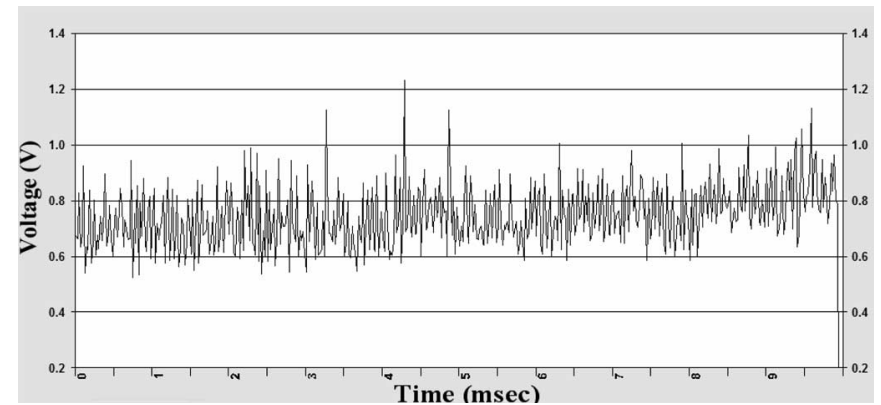

Fig. 8. Charge amplifier output over a period of $10 \mathrm{~ms}$, showing several large voltage peaks created by incoming neutrons.

period. Based on the estimated flux rate this threshold yields an effective detection efficiency of 5\%. Moreover, if the threshold is decreased by $0.1 \mathrm{~V}$, the efficiency approaches $20 \%$ as the partial charge sweeps induced by the geometry of the diode are also included in the detection counts. In addition, the waveform shows that the detected neutrons create voltage spikes that are noticeably larger in magnitude than the system's noise floor for threshold levels between 0.9 and $1 \mathrm{~V}$. For a $0.1-\mathrm{V}$ increase in the height of the voltage pulse, the calculation using the measured charge amplifier gain of $170 \mu \mathrm{V} / \mathrm{fC}$ indicates that there are approximately $3 \times 10^{6}$ more electrons involved. Thus, in this application, the counting and classification operation of the detector are not impacted by the system noise floor, and the probability of noise signals for this threshold range is negligible while significant detector efficiencies are achieved. Furthermore, the slight increasing trend of the baseline in Fig. 8 may be attributed to the saturation tendency of the charge-sensitive amplifier due to the possible arrival of neutrons in clusters.

Further confirmation that the system is detecting actual neutrons can be seen by examining the pulse height spectra measured by the detector. Pulse height spectra are commonly used in particle-detection applications. As the energy of a neutron is proportional to the content of its charge pulse, the height (amplitude) of the voltage pulse created by the charge-sensitive amplifier directly translates into detected energy. Energy levels are then quantized as channels, and detected particles are assigned to a channel based on their energy. The number of events in each channel is then plotted versus the number of channels. The pulse height spectra measured from the neutron source by the detector is shown in the top curve of Fig. 9(a). The ideal predicted spectra based on boron carbide neutrondetection characteristics is also plotted in Fig. 9(b).

The pulse height spectra was calibrated through a comparison with a conversion-layer device. These devices possess the same $\mathrm{Li}$ and $\mathrm{He}$ capture products as in reactions (1) and (2) and, hence, provide a means for energy calibration [1]-[3], [11]-[17]. In particular, the relative positions of the distinct energy peaks mentioned in Section II can be used as the calibration reference for the pulse height spectra. Thus, the predicted curve shows a generally decreasing energy spectrum with the exception of two distinct peaks occurring at 2.31 and $2.8 \mathrm{MeV}$ [20], [21]. This same general characteristic can also be seen in the measured spectra, with variations due to specific boron carbide diode properties that involve tailing and broadening due to incomplete charge collection and nonlinearities in the 


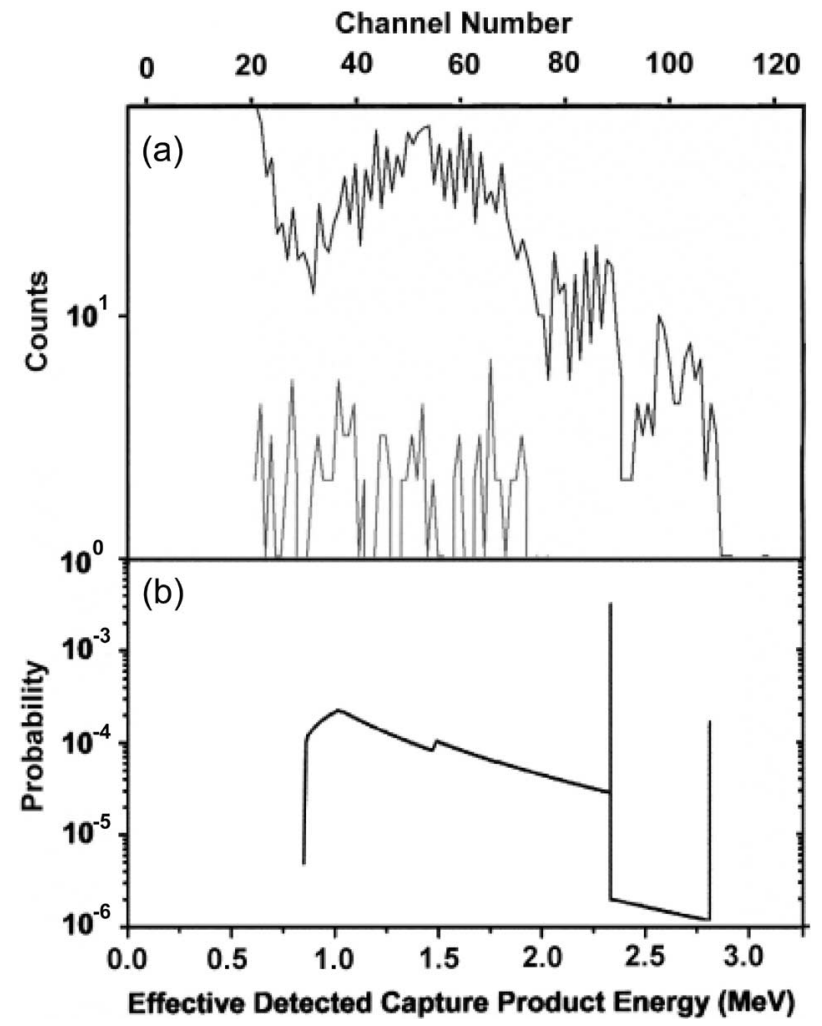

Fig. 9. Measured versus theoretical neutron pulse height spectra. The top curve in (a) is the measured pulse height spectra, and the theoretical pulse height spectra adapted from [20] is shown in (b) The bottom curve in (a) shows the much lower spectra that resulted when neutrons were blocked from the diode.

detection circuits. The sum peaks are not directly observed because of the finite energy resolution in experiment. Nonetheless, the neutron counts above $1 \mathrm{~V}$ level in Fig. 8 approximately correspond to where the energy sum peaks are located in the spectra, whereas a $0.1-\mathrm{V}$ drop in the level corresponds to the middle section of energy spectra. This is indicated roughly by a four times increase in the counts, which agrees with the increase in efficiency from $5 \%$ to $20 \%$ as previously mentioned.

While the energy sum peaks at 2.31 and $2.8 \mathrm{MeV}$ are not readily apparent in the measured spectra, their presence can be seen by examining the overall signal shaping of the system. The detection of neutrons by the boron carbide diode is affected by a number of random variables. The angle of incidence between the neutron and the diode can decrease the number of charges generated by the capture products. Total charge collection is not possible, as varying amounts of recombination can occur in the depletion region. Finally, the nonlinear gain of the charge amplifier can misrepresent the charge content of different pulses. In this paper, these uncertainties have been modeled as a probability density function (PDF) with a normal distribution that has a standard deviation of $\sigma=0.35 \mathrm{MeV}$.

Taking the ideal PDF shown in Fig. 9(b) and the PDF modeling nonidealities, the system that relates the two functions is shown in Fig. 10. The system relates the fact that the PDF of the sum of two independent random variables is equal to the convolution of their PDFs [27]. Thus, by convolving the ideal PDF, with the PDF modeling the random variables of the system, the energy spectra in the presence of random nonidealities can be determined.

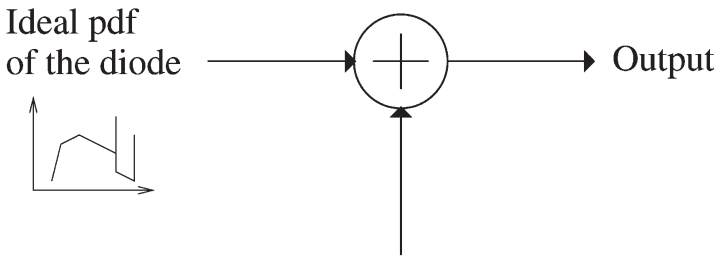

Amplitude variation pdf due to non-idealities

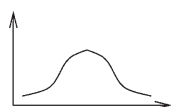

Fig. 10. Conceptual diagram of the system that relates the ideal neutron detection PDF with the nonideal effects of the detector.

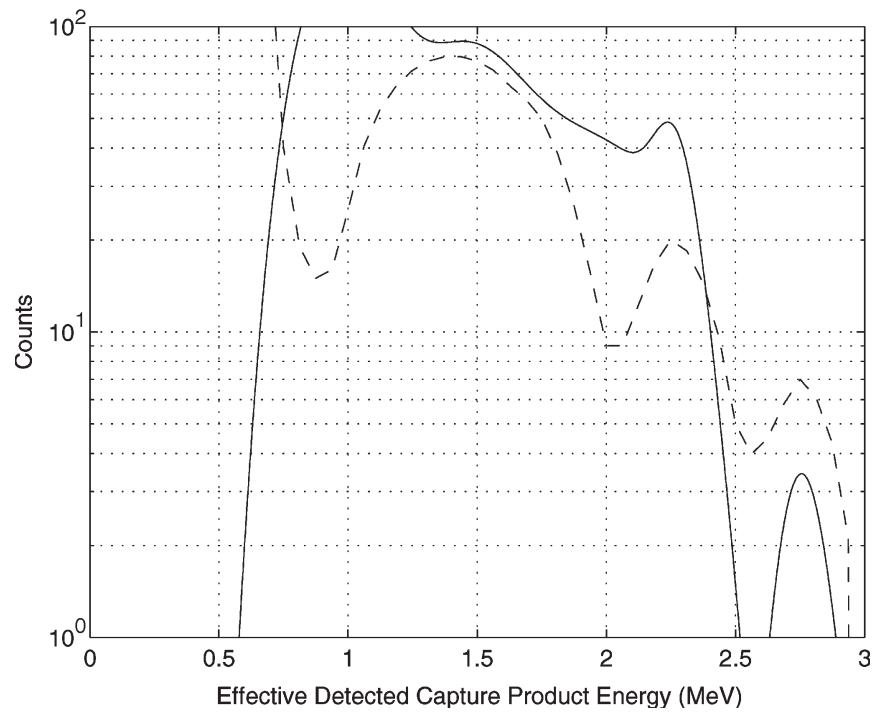

Fig. 11. Convolution-determined PDF (solid line) compared to an envelope waveform modeling the measured output of the handheld detector (dashed line).

The system was modeled in MATLAB by first recreating the ideal spectra of Fig. 9(b) and then generating the Gaussian PDF. It should be noted that the ideal spectrum was scaled by a factor of $10^{6}$ for convenience. When the two PDFs were convolved together, the resulting PDF is shown as the solid waveform in Fig. 11. The result shows that the random processes in the detection system cause the two ideal peaks to become much broader and lower. When this result is compared to a general envelope waveform modeling the observed output of Fig. 9(a), the observed peaks are a good match to the nonideal PDF. This can be seen in Fig. 11 where the measured output of the handheld detector is shown as the dashed line. The results show that neutrons are still being detected by the devices in the presence of the nonideal effects. The measured results then display this broadening of the 2.31- and $2.8-\mathrm{MeV}$ peaks based on these effects. It should be noted that this is a simple model of the complex interactions of the boron carbide diode and detector and certainly suffers from inaccuracies. The results clearly convey how the nonidealities of the diode and the system broaden and lower the sharp 2.31- and 2.8-MeV peaks. This model does match well with the measured pulse height spectra.

Another verification of the system's effectiveness at detecting neutrons can also be seen in the bottom curve Fig. 9(a). In this experiment, a 4-mm-thick cadmium foil was placed over the 
boron carbide diode. The cadmium foil blocks most incoming neutrons from the diode and resulted in the substantially lower spectra shown by the bottom curve. This result can be attributed to the small number of neutrons detected due to imperfect shielding of the cadmium foil. Finally, there were not any observed neutron events after the handheld detector was removed from the radiation source altogether, which validates the finding that there are no false detections.

\section{CONCLUSION}

This paper presented the overall design of a compact handheld neutron detector utilizing a new class of heteroisomeric boron carbide diode. The handheld operation was tested to verify that the heteroisomeric diode reacts with incoming neutrons and that the custom-designed charge amplifier front end microchip detects the resulting charge pulses and converts them to a voltage signal. The microcontroller architecture then samples that voltage signal at a very high rate. Real-time processing methods on the sampled signal can then classify the energy of incoming neutrons. While there is a discrepancy between the actual and theoretical energy spectra, the results of this paper demonstrate how the nonidealities of the diode and system broaden and lower the energy sum peaks. Consequently, this agrees well with the measured pulse height spectra for experimental results. Hence, the results presented here conclusively show that this new class of boron carbide semiconductor device is, indeed, an effective neutron detector. It has also been shown that the heteroisomeric class of all boron carbide diodes is distinct from both the heterojunction-diode and conversion-layer devices. The pulse height spectra differ from heterojunctiondiode and conversion-layer solid-state detectors because the depletion regions and both sides of the p-n junction contain a boron-rich semiconductor for neutron detection.

\section{REFERENCES}

[1] A. Mireshghi, G. Cho, J. Drewery, T. Jing, S. Kaplan, V. Perez-Mendez, and D. Wildermuth, "Amorphous silicon position sensitive neutron detector," IEEE Trans. Nucl. Sci., vol. 39, no. 4, pp. 635-640, Aug. 1992.

[2] B. Robertson, S. Adenwalla, A. Harken, P. Welsch, J. I. Brand, P. Dowben, and J. P. Claassen, "A class of boron-rich solid-state neutron detectors," Appl. Phys. Lett., vol. 80, no. 19, pp. 3644-3646, May 2002.

[3] S. Adenwalla, P. Welsch, A. Harken, J. Brand, A. Sezer, and B. Robertson, "Boron carbide/n-silicon carbide heterojunction diodes," Appl. Phys. Lett., vol. 79, no. 26, pp 4357-4359, Dec. 2001.

[4] S. Adenwalla, R. Billa, J. I. Brand, E. Day, M. J. Diaz, A. Harken, A. McMullen-Gunn, R. Padmanabhan, and B. W. Robertson, "Semiconducting boron-rich neutron detectors," in Proc. SPIE-Penetrating Radiation Systems and Applications V, F. P. Doty, H. B. Barber, and H. Roehrig, Eds., Dec. 2003, vol. 5199, pp. 70-74.

[5] Z. W. Bell, D. A. Carpenter, S. S. Cristy, V. E. Lamberti, A. Burger, B. F. Woodfield, T. Niedermayr, I. D. Hau, S. E. Labov, S. Friedrich, W. G. West, K. R. Pohl, and L. van der Berg, "Neutron detectors with cryogenics and semiconductors," Phys. Status Solidi, C, vol. 2, no. 5, pp. 1592-1605, 2005.

[6] A. Owens and A. Peacock, "Compound semiconductor radiation detectors," Nucl. Instrum. Methods Phys. Res. A, Accel. Spectrom. Detect. Assoc. Equip., vol. 531, no. 1/2, pp. 18-37, Sep. 2004.

[7] A. D. Harken, E. E. Day, B. W. Robertson, and S. Adenwalla, "Boronrich semiconducting boron carbide neutron detector," Jpn. J. Appl. Phys., vol. 44, no. 1A, pp. 444-445, 2005 .

[8] D. S. McGregor and J. K. Shultis, "Spectral identification of thin-filmcoated and solid-form semiconductor neutron detectors," Nucl. Instrum. Methods Phys. Res. A, Accel. Spectrom. Detect. Assoc. Equip., vol. 517, no. $1 / 2$, pp. 180-188, Jan. 2004.
[9] N. Sato, O. Ishiwata, Y. Seki, and A. Ueda, "Plasma CVD-grown ${ }^{10} \mathrm{~B}$-enriched boron films for Si neutron detectors," Jpn. J. Appl. Phys., vol. 29, no. 11, pp. 2526-2530, Nov. 1990.

[10] D. S. McGregor, S. M. Vernon, H. K. Gersch, S. M. Markham, S. J. Wojtczuk, and D. K. Wehe, "Self-biased boron-10 coated high-purity epitaxial GaAs thermal neutron detectors," IEEE Trans. Nucl. Sci., vol. 47 , no. 4, pp. 1364-1370, Aug. 2000.

[11] C. A. Baker, K. Green, M. G. D. van der Grinten, P. S. Iaydjiev, S. N. Ivanov, S. Al-Ayoubi, P. G. Harris, J. M. Pendlebury, D. B. Shiers, and P. Geltenbort, "Development of solid-state silicon devices as ultra cold neutron detectors," Nucl. Instrum. Methods Phys. Res. A, Accel. Spectrom. Detect. Assoc. Equip., vol. 487, no. 3, pp. 511-520, Jul. 2002.

[12] A. Rose, "Sputtered boron films on silicon surface barrier detectors," Nucl. Instrum. Methods, vol. 52, no. 1, pp. 166-170, Jun. 1967.

[13] B. W. Robertson, S. Adenwalla, A. Harken, P. Welsch, J. I. Brand, J. P. Claassen, N. M. Boag, and P. A. Dowben, "Semiconducting boronrich neutron detectors," in Proc. SPIE-Adv. Neutron Scattering Instrum., I. S. Anderson, and B. Guérard, Eds., 2002, vol. 4785, pp. 226-233.

[14] A. N. Caruso, R. B. Billa, S. Balaz, J. I. Brand, and P. A. Dowben, "The heteroisomeric diode," J. Phys. Condensed Matter, vol. 16, no. 10, pp. L139-L146, Mar. 2004.

[15] E. Day, M. J. Diaz, and S. Adenwalla, "Effect of bias on neutron detection in thin semiconducting boron carbide films," Appl. Phys. Lett., vol. 39, no. 14, pp. 2920-2924, Jul. 2006.

[16] D. Emin and T. L. Aselage, "A proposed boron carbide based solid-state neutron detector," J. Appl. Phys., vol. 97, no. 1, p. 013529, Jan. 2005.

[17] D. N. McIlroy, "Heteroisomeric diode-A solid state neutron detector," J. Phys. Condensed Matter, vol. 16, no. 18, pp. V13-V14, May 2004.

[18] S.-D. Hwang, D. Byun, N. J. Ianno, P. A. Dowben, and H. R. Kim, "Fabrication of boron carbide-Boron heterojunction devices," Appl. Phys. Lett., vol. 68, no. 11, pp. 1495-1497, Mar. 1996.

[19] S.-D. Hwang, K. Yang, P. A. Dowben, A. A. Ahmad, N. J. Ianno, J. Z. Li, J. Y. Lin, H. X. Jiang, and D. N. McIlroy, "Fabrication of n-type nickel doped $\mathrm{B} 5 \mathrm{C} 1$ + delta homojunction and heterojunction diodes," Appl. Phys. Lett., vol. 70, no. 8, pp. 1028-1030, Feb. 1997.

[20] A. D. Harken, C. N. Lundstedt, E. E. Day, and B. W. Robertson, "Neutron detection efficiency and capture product energy spectra of all-semiconducting-boron carbide and conversion-layer detectors," IEEE Trans. Nucl. Sci., submitted for publication.

[21] C. Lundstedt, A. Harken, E. Day, B. W. Robertson, and S. Adenwalla, "Modeling solid-state boron carbide low energy neutron detectors," Nucl. Instrum. Methods Phys. Res. A, Accel. Spectrom. Detect. Assoc. Equip., vol. 562, no. 1, pp. 380-388, Jun. 2006.

[22] A. N. Caruso, P. A. Dowben, S. Balkir, N. Schemm, K. Osberg, R. W. Fairchild, O. Barrios Flores, S. Balaz, A. D. Harken, B. W. Robertson, and J. I. Brand, "The all boron carbide diode neutron detector: Comparison with theory," Mater. Sci. Eng. B., vol. 135, no. 2, pp. 129-133, Nov. 2006.

[23] G. Bertuccio and A. Pullia, "A wide-band low-noise charge amplifier with high electron mobility transistor input stage," in Proc. IEEE Conf. Nucl. Sci. and Med. Imag., Nov. 1994, vol. 2, pp. 740-744.

[24] S. Tadja, J. Spiegel, and H. Williams, "A CMOS low noise and low power charge sampling integrated circuit for capacitive detector/sensor interfaces," IEEE J. Solid-State Circuits, vol. 30, no. 2, pp. 110-119, Feb. 1995 .

[25] Y. Hu, J. Solére, D. Lachartre, and R. Turchetta, "Design and performance of a low-noise, low-power consumption CMOS charge amplifier for capacitive detectors," IEEE Trans. Nuclear Sci., vol. 45, no. 1, pp. 119-123, Feb. 1998.

[26] Y. Hu, "High performance low noise charge preamplifier with DC coupling to particle silicon detectors in CMOS technology," Electron. Lett., vol. 34, no. 13, pp. 1274-1275, Jun. 1998.

[27] A. Papoulis, Probability, Random Variables, and Stochastic Processes. New York: McGraw-Hill, 1984, p. 135.

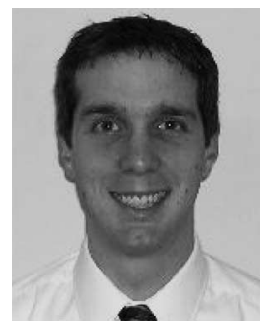

Kevin Osberg was born in Lincoln, NE, in 1980. He received the B.S. degree in computer engineering and the M.S. degree in electrical engineering from the University of Nebraska-Lincoln, in 2003 and 2005, respectively.

He is currently with LSI Logic Corporation, Fort Collins, CO. His research interests include analog and mixed-signal integrated circuit design for sensor applications, data converter, and amplifier design. 


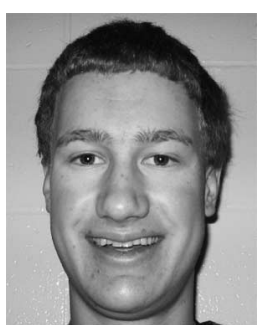

Nathan Schemm is currently working toward the B.S. degree in electrical engineering at the Department of Electrical Engineering, University of Nebraska-Lincoln.

His research interests include analog VLSI design and the integration and testing of such designs utilizing a microcontroller. Besides his work with the boron carbide diode, he is also interested in novel CMOS imager designs and applications, including work on various image-processing and compression circuits.

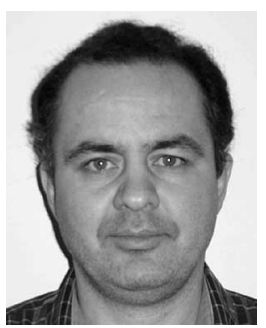

Sina Balkır received the B.S. degree in electrical engineering from Boğaziçi University, Istanbul, Turkey, in 1987 and the M.S. and Ph.D. degrees in electrical engineering from Northwestern University, Evanston, IL, in 1989 and 1992, respectively.

Between 1992 and 1998, he was with the Department of Electrical and Electronics Engineering, Boğaziçi University, as an Assistant and Associate Professor. He is currently with the Department of Electrical Engineering, University of NebraskaLincoln, where he serves as an Associate Professor. His research interests include CAD of VLSI systems, analog VLSI design automation, and focal plane computation arrays for image processing.

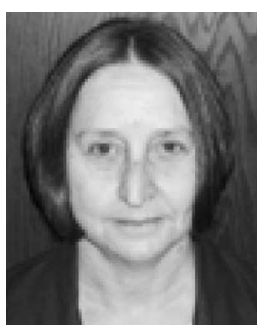

Jennifer I. Brand received the B.S. and M.S. degrees from the University of Michigan, Ann Arbor, and the Ph.D. degree from the University of California, San Diego.

A traditional chemical engineer with national laboratory and private sector work experience, she is currently a Professor with the College of Engineering and Technology, University of Nebraska-Lincoln. Her research focuses on materials processing for sensors and advanced energy systems.

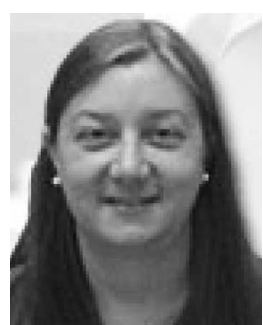

M. Susan Hallbeck received the B.S. degree in industrial engineering from the Iowa State University, Ames, in 1984, the M.S. degree in industrial engineering from Texas Tech University, Lubbock, in 1985, and the Ph.D. degree in industrial engineering and operations research from the Virginia Polytechnic Institute and State University, Blacksburg, in 1990.

She is currently a Professor with the Department of Industrial and Management Systems Engineering, University of Nebraska-Lincoln.

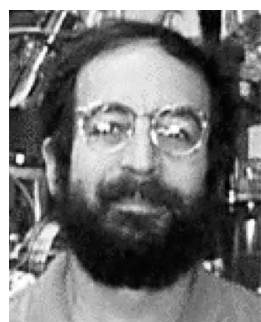

Peter A. Dowben received the B.A. degree in mathematics from Haverford College, Haverford, PA, in 1977 and the Ph.D. degree in physics from the University of Cambridge, Cambridge, U.K., in 1981.

After positions at the Free University of Berlin and the Fritz Haber Institute, Berlin, Germany, he joined the faculty of Syracuse University, Syracuse, NY. He is currently the Charles Bessey Professor of Physics and a Research Professor of chemistry with the Department of Physics and Astronomy, University of Nebraska-Lincoln.

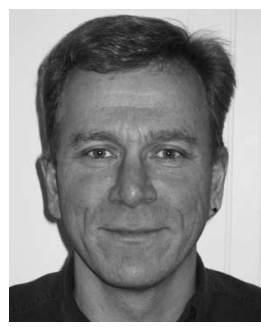

Michael W. Hoffman received the B.S. degree from Rice University, Houston, TX, the M.S. degree from the University of Southern California, Los Angeles, and the Ph.D. degree from the University of Minnesota, Twin Cities, all in electrical engineering.

From 1985 to 1988 , he was a Signal Processing System Engineer in the Space Communications Division, TRW Incorporated. In 1993, he joined the University of Nebraska-Lincoln, where he is currently an Associate Professor with the Department of Electrical Engineering. His research interests include data compression, joint source channel coding, and sensor array processing. 